\title{
Nuclear cardiology reporting: Leaving an impression
}

\author{
Edwin $\mathrm{Wu}, \mathrm{MD},{ }^{\mathrm{a}}$ and Thomas A. Holly, $\mathrm{MD}^{\mathrm{a}}$ \\ a Division of Cardiology, Northwestern University Feinberg School of Medicine, Chicago, IL
}

Received May 20, 2018; accepted May 21, 2018

doi:10.1007/s12350-018-1315-7

\section{See related article, pp. 1878-1885}

The performance and interpretation of myocardial perfusion imaging studies are obviously vital components of the stress testing process. How one reports the findings to the referring doctor and other healthcare providers is an all too important, and often neglected, part of the performance of any diagnostic test. Medical testing is frequently not binomial (normal/abnormal), but rather provides a mixture of current and future cardiovascular risk assessments for patients. The field of nuclear cardiology has a long history of publications highlighting these subtle differences to empower physicians to make appropriate and meaningful decisions while mitigating unnecessary procedures and decreasing exposure to potentially harmful tests and therapeutics. ${ }^{1-3}$ However, the quality of the information, both in imparting confidence in the accuracy of information and the expertise of the interpreting physician, is only as effective as the mode in which this information is communicated, which would be the imaging report.

Structured reporting can provide the necessary framework to communicate this information in a clear and succinct manner. ${ }^{4}$ However, the terminology of structured reporting can encompass a wide scope of models. ${ }^{5}$ Free-text voice or typed information can be inputted into a set of pre-defined headers. Other options include filling in the blanks with a selection of canned phrases, while other styles employ a standardized lexicon of medical terms within a rigid point-and-click system. Each reporting model has attributes that can

Reprint requests: Thomas A. Holly, MD, Division of Cardiology, Northwestern University Feinberg School of Medicine, Chicago, IL; tholly@nm.org

J Nucl Cardiol 2019;26:1886-7.

1071-3581/\$34.00

Copyright (C) 2018 American Society of Nuclear Cardiology. range from cumbersome to burdensome, and despite the potential benefits, physicians frequently cite the onerous work necessary to utilize any form of structure within their reports.

In this issue of the Journal, Levy et al. explore the possible use of Natural Language Processing (NLP) to determine ischemic risk assessment from stress myocardial perfusion imaging reports. The authors propose that NLP could be used to improve physicians' understanding of these results and improve utilization, specifically, proper utilization of cardiac catheterization and revascularization. However, before NLP automated algorithms can be employed to assist with data mining and translating text strings to assist with a final interpretation, all necessary clinical and medical data elements need to be present within the text report.

Levy et al. performed an analysis of myocardial perfusion imaging reports at VA medical centers to determine if an adequate assessment of ischemic risk can be ascertained from the wording in the reports. The investigators used a VA database to retrospectively identify patients who underwent cardiac catheterization and cardiac nuclear stress testing over a two-year period. Manual review of these reports by two of the authors found only $3 \%$ to $4 \%$ of the 99 reports they selected for analysis from 44 different VA stations explicitly stated an assessment of ischemic risk in the report. However, an assessment of the ischemic risk could be determined in $74 \%$ of the imaging reports when isolating and analyzing the "Impression" or "Summary" section of the imaging report. If the entire report was utilized to find all relevant data elements, the ability to assess and report the ischemic risk improved to nearly all $(98 \%)$ of the reports. What is clearly demonstrated in this publication is the need to standardize reporting structure in order to successfully extract data, describe findings, and improve a clinicians' understanding of the necessary clinical information of a nuclear stress report. ${ }^{6}$ 
What is the take home message from this paper? Perhaps the greatest priority, rather than machine learning or NLP, is to clearly state the important findings and overall impression in an imaging report. In our experience, clinicians frequently skim towards the end of reports, bypassing the details of any findings. While the desire to report risk is admirable, the question remains as to the type of risk: The risk of future myocardial infarction? Need for revascularization? Cardiac death or all-cause mortality? Levy and colleagues chose the path of identifying data elements to justify revascularization. While important and desirable to the interventional cardiologist (and probably the patient), this particular risk assessment is not the only use of myocardial perfusion imaging. What is clearly evident, however, is the need to be able to find the necessary information to make a clinical decision. Although components for a risk algorithm could be determined in $98 \%$ of the examined reports, the bar was set relatively low due to its narrow focus on ischemic risk as it relates to the need for revascularization. The analysis of the stress reports was limited to the documentation of the presence or absence of ischemia, a description of the ischemic lesion, if present, and a description of left ventricular systolic function. It was disheartening to find that only $42 \%$ of the impression sections and $34 \%$ of the entire reports did not comment on myocardial infarction, and that usage of a descriptive term such as a "fixed defect" was sometimes used instead of an interpretive finding such as "infarct" or "scar." It is also disappointing that the presence of myocardial ischemia had to be inferred from the descriptive wording ("reversible defect," for example) in $21 \%$ of the Impression sections. These descriptive and narrative terms of the nuclear images leave the interpretation to the imagination of any managing physicians, and reporting these terms in a final "Impression" is wholly vague, equivocal and, frankly, unacceptable. Moreover, the subtle quantitative differences imparting the severity of any fixed or reversible defect is missed, which can highlight differences in cardiac risks and event rates. Differences in the how this information is both presented and expressed can lead to confusion and perception of substandard clinical care. ${ }^{7}$ While structured reporting will not make that cease, developing structured reports which prompt or guide the interpreting physicians to include all data elements recommended in the current ASNC reporting guidelines is important to provide the critical information necessary to impart and communicate a clinically meaningful final impression. ${ }^{8}$

With the advent of electronic medical records, patient-related data have not only become more freely available, but sometimes increasingly abstruse. ${ }^{9}$
Structured reporting can aid in reporting findings that eventually are necessary to improve data collection, consistency in reporting, and quality assurance, as well as optimize patient care and promote patient safety. ${ }^{10}$ More than just facts, though, our reports should interpret these findings for clinicians. Natural language processing of reports may help us in the future, but for now, we should make certain to accurately describe and interpret the findings and appropriately communicate a clear impression of any appropriate risk to the referring provider.

\section{Disclosures}

All authors have nothing to disclose.

\section{References}

1. Hachamovitch R, Berman DS, Shaw LJ, Kiat H, Cohen I, Cabico JA, et al. Incremental prognostic value of myocardial perfusion single photon emission computed tomography for the prediction of cardiac death: differential stratification for risk of cardiac death and myocardial infarction. Circulation 1998;97(6):535-43.

2. Hachamovitch R. Comparison of the short-term survival benefit associated with revascularization compared with medical therapy in patients with no prior coronary artery disease undergoing stress myocardial perfusion single photon emission computed tomography. Circulation 2003;107(23):2900-7.

3. Doukky R, Hayes K, Frogge N, Balakrishnan G, Dontaraju VS, Rangel MO, et al. Impact of appropriate use on the prognostic value of single-photon emission computed tomography myocardial perfusion imaging. Circulation 2013;128(15):1634-43.

4. Schwartz LH, Panicek DM, Berk AR, Li Y, Hricak H. Improving communication of diagnostic radiology findings through structured reporting. Radiology 2011;260(1):174-81.

5. Gunderman RB, McNeive LR. Is structured reporting the answer? Radiology 2014;273(1):7-9.

6. Ghoshhajra BB, Lee AM, Ferencik M, Elmariah S, Margey RJP, Onuma $\mathrm{O}$, et al. Interpreting the interpretations: The use of structured reportingimproves referring clinicians'comprehension of coronary CT angiography reports. JACR 2013;10(6):432-8.

7. Trägårdh E, Höglund $\mathrm{P}$, Ohlsson $\mathrm{M}$, Wieloch $\mathrm{M}$, Edenbrandt L. Referring physicians underestimate the extent of abnormalities in final reports from myocardial perfusion imaging. EJNMMI Res 2012;2(1):27.

8. Tilkemeier PL, Bourque J, Doukky R, Sanghani R, Weinberg RL. ASNC imaging guidelines for nuclear cardiology procedures. Standardized reporting of nuclear cardiology procedures. J Nucl Cardiol 2017;24(6):2064-128.

9. Trägårdh E, Carlsson M, Edenbrandt L. Computerized decision making in myocardial perfusion SPECT: The new era in nuclear cardiology? J Nucl Cardiol 2015;22(5):885-7.

10. Douglas PS, Taylor A, Bild D, Bonow R, Greenland P, Lauer M, et al. Outcomes research in cardiovascular imaging: Report of a workshop sponsored by the National Heart, Lung, and Blood Institute. J Cardiovasc Comput Tomogr 2009;3(4):212-23. 